Tôhoku Math. Journ.

39 (1987), 447-464.

\title{
GEVREY HYPOELLIPTICITY OF A CLASS OF PSEUDODIFFERENTIAL OPERATORS
}

\author{
Dedicated to Professor Tadashi Kuroda on his sixtieth birthday
}

\section{Tadato Matsuzawa}

(Received September 4, 1985)

Introduction. We consider a pseudodifferential equation:

$$
a(x, D) u=f
$$

with data $f$ having Gevrey index $s \geqq 1$. Here $a(x, D)$ is a pseudodifferential operator of type $S_{\rho, \delta}^{m}$ of Hörmander (cf. [4]). We are interested in the Gevrey regularity of solutions, more precisely, in which way the Gevrey index of solutions depends on $\rho, \delta$ and $s$.

In [3], we have given the definition of a class of hypoelliptic pseudodifferential operators of symbol class $S_{\rho, \delta, \sigma}^{m}\left(\Omega \times R^{n}\right), \Omega \subset R^{n}, 0 \leqq \delta<\rho \leqq 1$, $\sigma \geqq 1$, which consists of symbols $a(x, \xi) \in S_{\rho, \delta}^{m}\left(\Omega \times R^{n}\right)$ satisfying

$$
\begin{aligned}
&|a(x, \xi)| \geqq c|\xi|^{m^{\prime}}, \quad|\xi| \geqq B,-\infty<m^{\prime}<\infty, \\
&\left|a_{(\beta)}^{(\alpha)}(x, \xi)\right| \leqq C_{0} C_{1}^{|\alpha+\beta|} \alpha ! \beta !^{\sigma}|a(x, \xi)|(1+|\xi|)^{-\rho|\alpha|+\delta|\beta|}, \\
& x \in \Omega,|\xi| \geqq B|\alpha|^{\theta}, \theta=\sigma /(\rho-\delta) .
\end{aligned}
$$

Under these conditions, we have constructed a parametrix $b$ of $a(x, D)$ with symbol $b(x, \xi) \in S_{\rho, \delta, a}^{-m^{\prime}}\left(\Omega \times R^{n}\right)$. Here $b$ is expressed by an infinite series of symbols, and the remainder $r=b a-I$ is an integral operator with a kernel of Gevrey function of index $\theta=\sigma /(\rho-\delta)$ (cf. Theorem 3.1 and Corollary 3.1 of [3]). Thus we have $\max (\sigma /(\rho-\delta), s)$ as the Gevrey index for solutions of the equation (1). This gives the best possible index when $\rho \equiv 1,0 \leqq \delta<1$ as was shown by several examples in [3], but not necessarily the best possible when $0<\rho<1$.

It seems impossible to apply directly the method of [3] to obtain sharper results if $0<\rho<1$. We use a finite approximation of parametrix instead of infinite approximation used in [3]. The remainder term is not necessarily smooth, so we are forced to estimate all derivatives of solutions inductively. This method seems unusual in the study of hypoellipticity because it looks tedious. However, surprisingly this method provides a sharper result for Gevrey hypoellipticity. For the nonlinear problem such a method was used by Friedman [2] to get 
analyticity of solutions of elliptic and parabolic systems and by Volevič [16] for a class of pseudodifferential equations.

Now we would like to summarize the basic idea of this paper partly motivated by [14] without going into technical details. We consider the equation (1) with $a(x, \xi)$ satisfying

$$
\begin{gathered}
|a(x, \xi)| \geqq c|\xi|^{m^{\prime}}, \quad|\xi| \geqq B, \\
\left|a_{(\beta)}^{(\alpha)}(x, \xi)\right| \leqq C_{0} C_{1}^{|\alpha+\beta|} \alpha ! B !^{\alpha}|a(x, \xi)|(1+|\xi|)^{-\rho|\alpha|+\delta|\beta|}, \quad x \in \Omega,|\xi| \geqq B .
\end{gathered}
$$

The condition $\left(3^{\prime}\right)$ is slightly stronger than (3) but enough for applications. We first construct a left parametrix $b^{N}(x, D)$ of $a(x, D)$ consisting of a finite number of pseudodifferential operators. Then we reduce the equation (1) into an integral equation.

$$
u=b^{N} f-r^{N} u, \quad\left(b^{N} a u=b^{N} f=u+r^{N} u\right) .
$$

By induction on $k=|\alpha|, k=0,1, \cdots$, we obtain successive estimates of type

$$
\sup _{x \in K \subset \subset \Omega}\left|D^{\alpha} u\right| \leqq C_{0} C_{1}^{|\alpha|} \alpha !^{\max (s, \theta)}, \quad \theta=\max (1 / \rho, \sigma /(1-\delta)),
$$

(cf. Theorem 3.1) .

Since we have $\sigma /(\rho-\delta)>\max (1 / \rho, \sigma /(1-\delta))$ for $0<\delta<\rho<1$ and $\sigma \geqq 1$, this improves our previous result of [3].

The plan of the paper is as follows. In $\S 1$, we start with the precise definition of pseudodifferential operators considered in this paper. The regularity properties of their kernels and the pseudolocal property will be studied. In $\S 2$, we shall consider the symbolic calculus of a composed operator. In $\S 3$, we shall prove the main result (Theorem 3.1) on Gevrey hypoellipticity of pseudodifferential equations. In $\S 4$, we shall give some examples of differential operators.

Finally we remark that the same problem has been investigated in [7] and [14] recently. In [7], similar results have been obtained by constructing parametrices for a class of degenerate parabolic pseudodifferential operators, and in [14] by applying the theory of multiple products of pseudodifferential operators. Compared with these results our proof given here would be significantly elementary.

The author would like to express his gratitude to Y. Morimoto for useful discussion and also to the referee who has given many valuable suggestions.

1. A class of pseudodifferential operators. Let $\Omega$ be an open subset of $R^{n}$ whose point is denoted by $x=\left(x_{1}, \cdots, x_{n}\right)$. We use general notation 
such as $|\alpha|=\alpha_{1}+\cdots+\alpha_{n}$ for a multi-index $\alpha=\left(\alpha_{1}, \cdots, \alpha_{n}\right)$ and $D^{\alpha}=$ $D_{1}^{\alpha_{1}} \cdots D_{n}^{\alpha_{n}}, D_{j}=1 / i \partial / \partial x_{j}, j=1, \cdots, n$, etc.

Definition 1.1. Let $u \in C^{\infty}(\Omega)$. Then we say that $u$ is in $G^{s}$ in $\Omega$ $(s \geqq 1)$ if for any compact set $K$ of $\Omega$ there are positive constants $C_{0}$ and $C_{1}$ such that

$$
\sup _{x \in \mathbb{K}}\left|D^{\alpha} u(x)\right| \leqq C_{0} C_{1}^{|\alpha|}|\alpha|^{|s| \alpha \mid}, \quad \alpha \in Z_{+}^{n} .
$$

Definition 1.2. Let $-\infty<m<\infty ; 0 \leqq \delta<\rho \leqq 1 ; \sigma \geqq 1$. We denote by $S_{\rho, \delta, \sigma}^{m}\left(\Omega \times R^{n}\right)$ the set of all $a(x, \xi) \in C^{\infty}\left(\Omega \times R^{n}\right)$ such that for every compact set $K$ of $\Omega$ there are positive constants $C_{0}, C_{1}$ and $B$ such that

$$
\sup _{x \in K}\left|a_{(\beta)}^{(\alpha)}(x, \xi)\right| \leqq C_{0} C_{1}^{|\alpha+\beta|} \alpha ! \beta !^{\sigma}|\xi|^{m-\rho|\alpha|+\delta|\beta|}, \quad|\xi| \geqq B,
$$

where $a_{(\beta)}^{(\alpha)}(x, \xi)=\partial_{\xi}^{\alpha} D_{x}^{\beta} a(x, \xi), \partial_{\xi}=\left(\partial / \partial \xi_{1}, \cdots, \partial / \partial \xi_{n}\right)$.

We associate with such a symbol $a(x, \xi)$ a pseudodifferential operator as usual:

$$
a(x, D) u(x)=(2 \pi)^{-n} \iint e^{i\langle x-y, \xi\rangle} a(x, \xi) u(y) d y d \xi, \quad u \in C_{0}^{\infty}(\Omega) .
$$

Let $K(x, y) \in \mathscr{D}^{\prime}(\Omega \times \Omega)$ be the distribution kernel of $a(x, D)$ expressed by the oscillatory integral:

$$
K(x, y)=(2 \pi)^{-n} \int e^{i\langle x-y, \xi\rangle} a(x, \xi) d \xi .
$$

The following theorems strengthen Theorem 1.1 in [3].

THEOREM 1.1. Let $a(x, \xi) \in S_{\rho, \delta, o}^{m}\left(\Omega \times R^{n}\right)$. Then we have

$$
K(x, y) \in G_{x, y}^{\theta_{0}, 1 / \rho}(\Omega \times \Omega \backslash \Delta), \quad \Delta=\{(x, x) ; x \in \Omega\}, \quad \theta_{0}=\max (1 / \rho, \sigma+\delta / \rho) \text {. }
$$

THEOREM 1.2. If $u \in \mathscr{E}^{\prime}(\Omega)$ which is in $G^{s}(s \geqq 1)$ in a neighborhood of $x_{0} \in \Omega$, then we have $a(x, D) u \in G^{\theta_{1}}$ in the same neighborhood of $x_{0} \in \Omega$, where $\theta_{1}=\max (s, \sigma+s \delta, 1 / \rho, \sigma+\delta / \rho)$. More precisely, we have

$$
\begin{array}{ll}
\theta_{1}=\max (1 / \rho, \sigma+\delta / \rho) & \text { if } 1 \leqq s \leqq \min (1 / \rho, \sigma /(1-\delta)) ; \\
\theta_{1}=\sigma+s \delta & \text { if } 1 / \rho \leqq s \leqq \sigma /(1-\delta) ; \\
\theta_{1}=1 / \rho & \text { if } \sigma /(1-\delta) \leqq s \leqq 1 / \sigma ; \\
\theta_{1}=s & \text { if } s \geqq \max (1 / \rho, \sigma /(1-\delta)) .
\end{array}
$$

Proof OF THEOREM 1.1. Let $U$ be any compact set of $\Omega \times \Omega \backslash \Delta$. For each $(x, y) \in U$ and $\alpha, \beta \in Z_{+}^{n}$, we have in the sense of oscillatory integral: 


$$
D_{x}^{\alpha} D_{y}^{\beta} K(x, y)=(2 \pi)^{-n} \sum_{\gamma+\tau=\alpha} \frac{\alpha !}{\gamma ! \tau !} \int e^{i\langle x-y, \xi\rangle} \xi^{\gamma}(-\xi)^{\beta} a_{(\tau)}(x, \xi) d \xi .
$$

We have the estimate

$$
\left|\int_{|\xi| \leqq B} e^{i\langle x-y, \xi\rangle} \xi^{\gamma}(-\xi)^{\beta} a_{(\tau)}(x, \xi) d \xi\right| \leqq C_{0} C_{1}^{|\alpha+\beta|} \tau !^{\sigma}
$$

with constants $C_{0}$ and $C_{1}$ independent of $\alpha, \beta$ and $\tau \leqq \alpha$. Next, by setting

$$
N=\left[\left(|\gamma+\beta|+\delta|\tau|+m_{+}+n+2\right) / \rho\right], \quad m_{+}=\max (m, 0),
$$

we have for a fixed $i, 1 \leqq i \leqq n$,

$$
\begin{aligned}
\left(x_{i}-\right. & \left.y_{i}\right)^{N} \int_{|\xi| \geqq B} e^{i\langle x-y, \xi\rangle} \xi^{\gamma}(-\xi)^{\beta} a_{(\tau)}(x, \xi) d \xi \\
= & \int_{|\xi| \geqq B} e^{i\langle x-y, \xi\rangle} D_{\xi_{i}}^{N}\left\{\xi^{\gamma}(-\xi)^{\beta} a_{(\tau)}(x, \xi)\right\} d \xi \\
& \quad-\sum_{k=0}^{N-1}\left(x_{i}-y_{i}\right)^{N-1-k} \int_{|\xi|=B} e^{i\langle x-y, \xi\rangle} D_{\xi_{i}}^{k}\left\{\xi^{\gamma}(-\xi)^{\beta} a_{(\tau)}(x, \xi)\right\} d S_{\xi} \\
\equiv & G_{1}(x, y)-G_{2}(x, y) .
\end{aligned}
$$

By the hypothesis (1.2) the integrand of $G_{1}$ is estimated by

$$
\begin{aligned}
& \mid \sum_{k=0}^{\gamma_{i}+\beta_{i}} \frac{N !\left(\gamma_{i}+\beta_{i}\right) !}{k !(N-k) !\left(\gamma_{i}+\beta_{i}-k\right) !^{\gamma+\beta} \xi_{i}^{-k} D_{\xi_{i}}^{N-k} a_{(\tau)}(x, \xi) \mid} \\
& \quad \leqq C_{0} C_{1}^{|\tau|+N}(\tau !)^{\sigma} N ! \sum_{k=0}^{\gamma_{i}+\beta_{i}}\left(\begin{array}{c}
\gamma_{i}+\beta_{i} \\
k
\end{array}\right)|\xi|^{|\gamma+\beta|-k}|\xi|^{m_{+}-\rho(N-k)+\delta|\tau|} .
\end{aligned}
$$

Taking another couple of constants $C_{0}$ and $C_{1}$ we can estimate this by

$$
C_{0} C_{1}^{|\alpha+\beta|} \tau !^{o} N !(1+|\xi|)^{-n-1} \text {. }
$$

By the definition of the number $N$, we have

$$
\tau !^{\sigma} N ! \leqq C^{|\alpha+\beta|}|\beta|^{(|\beta| / \rho)}|\gamma|^{(|\gamma| / \rho)}|\tau|^{(\sigma+\delta / \rho)|\tau|}
$$

with a constant $C$ independent of $\alpha, \beta$ and $\tau \leqq \alpha$. Hence we have an estimate of type

$$
\left|G_{1}(x, y)\right| \leqq C_{0} C_{1}^{|\alpha+\beta|}|\alpha|^{\theta_{0}|\alpha|}|\beta|^{(|\beta| / \rho)}, \quad \theta_{0}=\max (1 / \rho, \sigma+\delta / \rho) .
$$

Similar estimate holds for $G_{2}(x, y)$. Since $U$ is a compact set of $\Omega \times \Omega \backslash \Delta$, one can find a direction $i, 1 \leqq i \leqq n$, such that $\left|x_{i}-y_{i}\right| \geqq d>0$ for any $(x, y) \in U$ and finally we have the estimate

$$
\sup _{(x, y) \in U}\left|D_{x}^{\alpha} D_{y}^{\beta} K(x, y)\right| \leqq C_{0} C_{1}^{|\alpha+\beta|}|\alpha|^{\theta_{0}|\alpha|}|\beta|^{(|\beta| / \rho)}, \quad \alpha, \beta \in Z_{+}^{n},
$$

where the constants $C_{0}$ and $C_{1}$ are independent of $\alpha$ and $\beta$. 
Proof of Theorem 1.2. We first remark that for $f \in C_{0}^{\infty}\left(\Omega^{\prime}\right), \Omega^{\prime}$ is a relatively compact open subset of $\Omega$, and we have

$$
\begin{aligned}
\left|D_{x}^{\alpha}\{a(x, D) f(x)\}\right| \leqq \sum_{\gamma+\tau=\alpha}\left(\begin{array}{l}
\alpha \\
\gamma
\end{array}\right) C^{|\tau|+1}(\tau !)^{\sigma} \operatorname{Vol}\{\operatorname{supp} f\} \\
\quad \times \sup _{x \in \Omega}\left|D_{x}^{r}(1-\Delta)^{N} f(x)\right|,
\end{aligned}
$$

where the constant $C$ depends only on $\Omega^{\prime}$ and $N=N(\tau)=\left[\left(\delta|\tau|+m_{+}+\right.\right.$ $n+2) / 2$ ]. Indeed, we have

$$
\begin{aligned}
& D_{x}^{\alpha} a(x, D) f(x)=(2 \pi)^{-n} \sum_{\gamma+\tau=\alpha}\left(\begin{array}{l}
\alpha \\
\gamma
\end{array}\right) \iint e^{i\langle x-y, \xi\rangle} \xi^{\gamma} a_{(\tau)}(x, \xi) f(y) d y d \xi \\
& \quad=(2 \pi)^{-n} \sum_{r+\tau=\alpha}\left(\begin{array}{l}
\alpha \\
\gamma
\end{array}\right) \iint e^{i\langle x-y, \xi\rangle}\left(1+|\xi|^{2}\right)^{-N} a_{(\tau)}(x, \xi)\left(1-\Delta_{y}\right)^{N} D_{y}^{r} f(y) d y d \xi
\end{aligned}
$$

which gives the estimate (1.7).

Now we take $u \in \mathscr{E}^{\prime}(\Omega)$ which is in $G^{s}$ in a bounded neighborhood $V$ of $x_{0} \in \Omega$. Let $U$ be a neighborhood of $x_{0}$ such that $\bar{U} \subset V$. There is a positive number $d$ such that $0<d<\operatorname{dis}\left(U, R^{n} \backslash V\right)$. Let $\left\{g_{l}\right\}_{l=0}^{\infty}$ be a series of functions in $C_{0}^{\infty}(V)$ such that $g_{l}(x)=1$ on $\{x ; \operatorname{dis}(x, U)<d\}$ and $\left|D_{x}^{\alpha} g_{l}(x)\right| \leqq C^{l} \alpha$ ! if $|\alpha| \leqq l$, where the constant $C$ is independent of $l$ (cf. [5]). Then we have for $x \in U$

$$
D_{x}^{\alpha}\{a(x, D) u(x)\}=D_{x}^{\alpha}\left\{a(x, D) g_{l} u(x)\right\}+D_{x}^{\alpha} \int K(x, y)\left\{1-g_{l}(y)\right\} u(y) d y .
$$

By using (1.7) we have

$$
\left|D_{x}^{\alpha} a(x, D) g_{l} u(x)\right| \leqq \sum_{\gamma+\tau=\alpha}\left(\begin{array}{l}
\alpha \\
\gamma
\end{array}\right) C^{|\tau|+1}(\tau !)^{o} \sup _{x \in \Omega}\left|D_{x}^{\gamma}(1-\Delta)^{N}\left\{g_{l}(x) u(x)\right\}\right|,
$$

where $N=\left[\left(\delta|\tau|+m_{+}+n+2\right) / 2\right]$. Taking $l=2|\alpha|$ we have

$$
\left|D_{x}^{\alpha} a(x, D) g_{l} u(x)\right| \leqq C_{1}^{|\alpha|+1} \sum_{\gamma+\tau=\alpha}|\tau|^{(\sigma+s \delta)|\tau|}|\gamma|^{s|\gamma|} \leqq C_{2}^{|\alpha|+1}|\alpha|^{\max (8, \sigma+8 \delta)|\alpha|}
$$

By Theorem 1.1, the last term of $(1.8)$ is in $G^{\theta_{0}}$ in $U, \theta_{0}=\max (1 / \rho, \sigma+\delta / \rho)$. Thus $a(x, D) u$ is in $G^{\theta_{1}}$ in $U$, where $\theta_{1}=\max (s, \sigma+s \delta, 1 / \rho, \sigma+\delta / \rho)$.

We only verify the case (4), the other cases being treated similarly. Namely, we assume $s \geqq \max (1 / \rho, \sigma /(1-\delta))$. Then we have $s \geqq \sigma+s \delta$ and $s \geqq \sigma+\delta / \rho$, which proves the assertion (4).

2. Symbolic calculus. Let $a(x, \xi) \in S_{\rho, \delta, \theta}^{m^{\prime}}\left(\Omega \times R^{n}\right)$ and $b(x, \xi) \in S_{\rho, \delta, \sigma}^{m^{\prime \prime}}(\Omega \times$ $\left.R^{n}\right)$. Let $\Omega^{\prime}$ be a relatively compact open subset of $\Omega$ and take $h \in C_{0}^{\infty}(\Omega)$ so that $h=1$ on a neighborhood $\widetilde{U}$ of $\bar{\Omega}^{\prime}$. Then the symbol of the operator $r(x, D)=a(x, D) h b(x, D)$ is given by 


$$
\begin{aligned}
r(x, \xi) & =a(x, D+\xi) h(x) b(x, \xi) \\
& =(2 \pi)^{-n} \int_{R_{\eta}^{n}} \int_{\Omega} e^{i\langle x-y, \eta\rangle} a(x, \xi+\eta) h(y) b(y, \xi) d y d \eta
\end{aligned}
$$

We set

$$
r^{N}(x, \xi)=\sum_{|\alpha| \leqq N} \frac{1}{\alpha !} a^{(\alpha)}(x, \xi) b_{(\alpha)}(x, \xi), \quad N=0,1, \cdots
$$

Then we easily see $r^{N}(x, \xi) \in S_{\rho, \delta, o}^{m}\left(\Omega \times R^{n}\right), m=m^{\prime}+m^{\prime \prime}, N=0,1, \cdots$.

THEOREM 2.1. We have

$$
r(x, D)=r^{N}(x, D)+F^{N}(x, D) \text { in } \Omega^{\prime},
$$

where $F^{N}(x, D)$ can be written as a sum of two operators, $F^{N}(x, D)=$ $F_{1}^{N}+F_{2}^{N} . \quad F_{1}^{N}$ is an integral operator from $C^{\infty}\left(\bar{\Omega}^{\prime}\right)$ into $G^{\theta}\left(\Omega^{\prime}\right)$ with kernel $F_{1}^{N}(x, y),(x, y) \in\left(\Omega_{x}^{\prime} \times \Omega_{y}^{\prime}\right), \theta=\max (1 / \rho, \sigma /(1-\delta)) . \quad F_{2}^{N}$ is a pseudodifferential operator with symbol $F_{2}^{N}(x, \xi)$ satisfying the condition

$$
\begin{aligned}
\left|D_{x}^{\beta} \partial_{\xi}^{\gamma} F_{2}^{N}(x, \xi)\right| \leqq & C_{0} C_{1}^{N+|\beta+\gamma|} N !^{\sigma} \gamma ! \beta !^{\sigma}|\xi|^{m_{+}+n-(\rho-\delta) N-\rho|\gamma|} \\
& \times \sum_{\tau \leqq \beta}\left(\begin{array}{c}
\beta \\
\tau
\end{array}\right)|\tau|^{\sigma \delta|\tau|}|\xi|^{\delta|\beta-\tau|+\delta^{2}|\tau|} \\
m_{+}= & \max (m, 0), x \in \Omega^{\prime},|\xi| \leqq B \quad \text { (cf. (1.3)). }
\end{aligned}
$$

More precisely, we can write

$$
D_{x}^{\beta} F_{2}^{N}(x, \xi)=\sum_{\tau \leqq \beta}\left(\begin{array}{l}
\beta \\
\tau
\end{array}\right) u^{\tau}(x, \xi),
$$

where each $u^{\tau}(x, \xi)$ satisfies an estimate of type

$$
\begin{array}{r}
\left|\partial_{\xi}^{\gamma} u^{\tau}(x, \xi)\right| \leqq C_{0} C_{1}^{N+|\gamma+\beta|} N !^{\sigma} \gamma ! \beta !^{\sigma} \tau !^{\sigma \delta}|\xi|^{m_{+}+n-(\rho-\delta) N-\rho|\gamma|+\delta|\beta-\tau|+\delta^{2}|\tau|}, \\
x \in \Omega^{\prime}, \quad|\xi| \geqq B .
\end{array}
$$

Proof. First we choose cut-off functions $h_{l}(x) \in C_{0}^{\infty}(\Omega),=1$ on the neighborhood $\widetilde{U}$ of $\bar{\Omega}^{\prime}$, with support in a fixed compact set. Moreover, we assume

$$
\left|D^{\alpha} h_{l}(x)\right| \leqq C^{l} \alpha !, \quad|\alpha| \leqq l+m_{+}^{\prime}+n+2, \quad l=0,1, \cdots,
$$

where the constant $C$ is independent of $l$. We express $F(x, \xi)=r(x, \xi)-$ $r^{N}(x, \xi)$ by

$$
\begin{aligned}
F^{N}(x, \xi) & =r(x, \xi)-r_{l}(x, \xi)+r_{l}(x, \xi)-r_{l}^{N}(x, \xi)+r_{l}^{N}(x, \xi)-r^{N}(x, \xi) \\
& =r_{l}^{1}(x, \xi)+r_{l}^{2}(x, \xi)+r_{l}^{3}(x, \xi),
\end{aligned}
$$

where $r_{l}(x, \xi)=a(x, D+\xi) h_{l}(x) b(x, \xi)$ and 


$$
r_{l}^{N}(x, \xi)=\sum_{|\alpha| \leq N} \frac{1}{\alpha !} a^{(\alpha)}(x, \xi) D_{x}^{\alpha}\left\{h_{l}(x) b(x, \xi)\right\} .
$$

We note that $r_{i}^{3}(X, \xi) \equiv 0$ in $\Omega^{\prime} \times R^{n}$. Let $K_{a}(x, y)$ and $K_{b}(x, y) \in \mathscr{D}^{\prime}(\Omega \times \Omega)$ be the distribution kernels of $a(x, D)$ and $b(x, D)$, respectively. Then for $u \in C_{0}^{\infty}(\Omega)$ we have

$$
\begin{aligned}
r_{l}^{1}(x, D) u(x) & =\int K_{a}(x, z)\left\{h(z)-h_{l}(z)\right\}\left\{\int K_{b}(z, y) u(y) d y\right\} d z \\
& =\int\left(\int K_{a}(x, z)\left\{h(z)-h_{l}(z)\right\} K_{b}(z, y) d z\right) u(y) d y .
\end{aligned}
$$

Hence the kernel of $r_{l}^{1}(x, D)$ is given by

$$
K_{l}^{1}(x, y)=\int_{\Omega} K_{a}(x, z)\left\{h(z)-h_{l}(z)\right\} K_{b}(z, y) d z .
$$

This is in $G_{x, y}^{\theta_{0}, 1 / \rho}$ in $\Omega^{\prime} \times \Omega^{\prime}$ uniformly with respect to $h_{l}, l=0,1, \cdots$, (cf. Theorem 1.1), and so we shall use this as if it is not depending on $l$.

Next we observe $r_{l}^{2}(x, \xi)$. We have for $x, \xi \in \Omega^{\prime} \times R^{n}$

$$
r_{l}^{2}(x, \xi)=(2 \pi)^{-n} \iint e^{i\langle x-y, \eta\rangle}\left[a(x, \xi+\eta)-\sum_{|\alpha| \leqslant N} \frac{1}{\alpha !} a^{(\alpha)}(x, \xi) \eta^{\alpha}\right] u(y, \xi) d y d \eta,
$$

where we have written $u(y, \xi)=h_{l}(y) b(y, \xi)$. We shall need the following cut-off functions $\chi_{j}(\xi) \in C_{0}^{\infty}\left(R^{n}\right), j=0,1, \cdots$, such that $\chi_{j}(\xi)=1$ for $|\xi| \leqq$ $1 / 4, \chi_{j}(\xi)=0$ for $|\xi| \geqq 1 / 2$ and $\left|\chi_{j}^{(\alpha)}(\xi)\right| \leqq C^{j} \alpha$ ! for $|\alpha| \leqq j+1$, where the constant $C$ is independent of $j, j=0,1, \ldots$. By using $\chi_{j}(\xi)$ we divide $r_{l}^{2}(x, \xi)$ into four parts:

$$
\begin{aligned}
r_{l}^{2}(x, \xi)= & (2 \pi)^{-n} \iint e^{i\langle x-y, \eta\rangle}\left[a(x, \xi+\eta)-\sum_{|\alpha| \leq N} \frac{1}{\alpha !} a^{(\alpha)}(x, \xi) \eta^{\alpha}\right] \chi_{j}\left(\frac{\eta}{|\xi|}\right) u(y, \xi) d y d \eta \\
& +(2 \pi)^{-n} \sum_{|\alpha| \leq N} \frac{1}{\alpha !} a^{(\alpha)}(x, \xi) \iint e^{i\langle x-y, \eta\rangle} \eta^{\alpha}\left[\chi_{j}\left(\frac{\eta}{|\xi|}\right)-1\right] u(y, \xi) d y d \eta \\
& +(2 \pi)^{-n} \iint e^{i\langle x-y, \eta\rangle} a(x, \xi+\eta)\left[1-\chi_{j}\left(\frac{\eta}{|\xi|}\right)\right]\left[1-\chi_{j}\left(\frac{\xi+\eta}{|\xi|}\right)\right] u(y, \xi) d y d \eta \\
& +(2 \pi)^{-n} \iint e^{i\langle x-y, \eta\rangle} a(x, \xi+\eta)\left[1-\chi_{j}\left(\frac{\eta}{|\xi|}\right)\right] \chi_{j}\left(\frac{\xi+\eta}{|\xi|}\right) u(y, \xi) d y d \eta \\
= & I_{1}(x, \xi)+I_{2}(x, \xi)+I_{3}(x, \xi)+I_{4}(x, \xi) .
\end{aligned}
$$

Concerning $I_{1}(x, \xi)$, we have

$$
\begin{aligned}
D_{x}^{\beta} \partial_{\xi}^{\gamma} I_{1}(x, \xi)= & (2 \pi)^{-n} \sum_{\substack{\tau \leq \beta \\
\mu \leq r}}\left(\begin{array}{c}
\beta \\
\tau
\end{array}\right)\left(\begin{array}{c}
\gamma \\
\mu
\end{array}\right) \iint e^{i\langle x-y, \eta\rangle} \sum_{|\alpha|=N+1} \frac{N+1}{\alpha !} \int_{0}^{1}(1-t)^{N} \\
& \times \alpha_{(\tau)}^{(\alpha+\mu)}(x, \xi+t \eta) d t D_{y}^{\alpha+\beta-\tau} \partial_{\xi}^{r-\mu}\left[\chi_{j}\left(\frac{\eta}{|\xi|}\right) u(y, \xi)\right] d y d \xi .
\end{aligned}
$$


We have $|\xi| / 2 \geqq|\xi+t \eta| \leqq 3|\xi| / 2$ and $|\eta| \leqq|\xi| / 2$ when $\chi_{j}(\eta /|\xi|) \neq 0$ and $0 \leqq t \leqq 1$. By using this and taking $j=N+|\gamma|+1$ and $l=N+|\beta|$, we have an estimate of type

(2.5) $\left|D_{x}^{\beta} \partial_{\xi}^{\gamma} I_{1}(x, \xi)\right| \leqq C_{0} C_{1}^{N+|\beta+\gamma|} N ! \gamma ! \beta !^{o}\left|\xi^{|m+n-(\rho-\delta) N-\rho| \gamma|+\delta| \beta \mid}, \quad x \in \Omega^{\prime}, \quad\right| \xi \mid \geqq B$. Next we consider $I_{2}(x, \xi)$. We have

$$
\begin{aligned}
& D_{x}^{\beta} \partial_{\xi}^{\gamma} I_{2}=\sum_{|\alpha| \leq N} \sum_{\substack{\tau \leq \beta \\
\mu \leq \gamma}}\left(\begin{array}{l}
\beta \\
\tau
\end{array}\right)\left(\begin{array}{l}
\gamma \\
\mu
\end{array}\right) \frac{1}{\alpha !} a_{(\tau)}^{(\alpha+\mu)}(x, \xi)\left[\int u_{(\alpha+\beta-\tau)}^{(\gamma-\mu)}\left(x-\frac{y}{|\xi|}, \xi\right) \chi_{j}(y) d y\right. \\
& \left.-u_{(\alpha+\beta-\tau)}^{(\gamma-\mu)}(x, \xi)\right] \\
& +\sum_{|\alpha| \leq N} \sum_{\substack{\tau \leq \beta \\
\mu \leq \gamma}}\left(\begin{array}{c}
\beta \\
\tau
\end{array}\right)\left(\begin{array}{c}
\gamma \\
\mu
\end{array}\right) \frac{1}{\alpha !} a_{(\tau)}^{(\alpha+\mu)}(x, \xi) \sum_{\substack{\lambda \leq \gamma-\mu \\
\lambda \neq 0}}\left(\begin{array}{c}
\gamma-\mu \\
\lambda
\end{array}\right) \int e^{i\langle x-y, \eta\rangle} u_{(\alpha+\beta-\lambda)}^{(\gamma-\mu-\lambda)}(y, \xi) \\
& \times \partial_{\xi}^{\lambda} \chi_{j}\left(\frac{\eta}{|\xi|}\right) d y d \eta
\end{aligned}
$$

where

$$
\chi_{j}(x)=(2 \pi)^{-n} \int e^{i\langle x, \eta\rangle} \chi_{j}(\eta) d \eta
$$

Noting that $\int \chi_{j}(x) d x=\chi_{j}(0)=1$ and that the other moment of $\chi_{j}$ is equal to zero, we see that the first sum on the right hand side is equal to

$$
\begin{aligned}
\sum_{|\alpha| \leq N} \sum_{\tau \leq \beta}\left(\begin{array}{l}
\beta \\
\tau \leq \gamma
\end{array}\right)\left(\begin{array}{l}
\gamma \\
\tau
\end{array}\right) \frac{1}{\alpha !} a_{(\tau)}^{(\alpha+\mu)}(x, \xi) \sum_{|\kappa|=N+1-|\alpha|} \frac{N+1-\alpha}{\kappa !} \int(y /|\xi|)^{\kappa} \\
\\
\times u_{(\alpha+\beta-\tau+\kappa)}^{(\gamma-\mu)}(x-t y /|\xi|, \xi)(1-t)^{N-|\alpha|+1} d t \chi_{j}(y) d y .
\end{aligned}
$$

We have by the definition of $\chi_{j}, j=N+|\gamma|+1$,

$$
\int\left|y^{\alpha} \chi_{j}(y)\right| d y \leqq C^{N+|r|+1} \alpha !,|\alpha| \leqq N+1 .
$$

We recall that we have taken $l=N+|\beta|$. Then we have $|\alpha+\beta-\tau+\kappa| \leqq$ $N+|\beta|+1$ and noting that $\operatorname{supp} \partial_{\xi}^{2} \chi_{j}(\eta /|\xi|) \subset\{|\xi| / 4 \leqq|\eta| \leqq|\xi| / 2\}, \lambda \neq 0$, we have finally

$$
\left|D_{x}^{\beta} \partial_{\xi}^{\gamma} I_{2}\right| \leqq C_{0} C_{1}^{N+|\alpha+\beta|} N !^{\sigma} \gamma ! \beta !^{\sigma}|\xi|^{m-(\rho-\delta) N-\rho|\gamma|+\delta|\beta|}, \quad x \in \Omega^{\prime}, \quad|\xi| \geqq B,
$$

where the constants $C_{0}$ and $C_{1}$ are independent of $N, \beta$ and $\gamma$.

Now we consider $I_{3}(x, \xi)$. We have

$$
\begin{aligned}
D_{x}^{\beta} \partial_{\xi}^{\gamma} I_{3}(x, \xi)= & (2 \pi)^{-n} \sum_{\substack{\tau \leq \beta \\
\mu \_\gamma}}\left(\begin{array}{c}
\beta \\
\tau
\end{array}\right)\left(\begin{array}{c}
\gamma \\
\mu
\end{array}\right) \iint e^{i\langle x-y, \eta\rangle} a_{(\tau)}^{(\mu)}(x, \xi+\eta) \\
& \times \partial_{\xi}^{\gamma-\mu}\left\{\left[1-\chi_{j}\left(\frac{\eta}{|\xi|}\right)\right] \cdot\left[1-\chi_{j}\left(\frac{\xi+\eta}{\xi}\right)\right] u_{(\beta-\tau)}(y, \xi)\right\} d y d \eta
\end{aligned}
$$




$$
\begin{array}{r}
=(2 \pi)^{-n} \sum_{\substack{\tau \leq \beta \\
\mu \leq \gamma \\
\lambda \leq \gamma-\rho}}\left(\begin{array}{c}
\beta \\
\tau
\end{array}\right)\left(\begin{array}{c}
\gamma \\
\mu
\end{array}\right)\left(\begin{array}{c}
\gamma-\mu \\
\lambda
\end{array}\right) \sum_{\substack{|\alpha|=N+, m_{+}+n+2 \\
=: N^{\prime}}} \frac{N^{\prime} !}{\alpha !} \int H_{\alpha, \mu, \tau, \lambda}(x, x-y, \xi) \\
\times\left(-\Delta_{y}\right)^{[\delta|\tau| / 2]} u_{(\alpha+\beta-\tau)}^{(\lambda)}(y, \xi) d y,
\end{array}
$$

where

$$
\begin{aligned}
H_{\alpha, \mu, \tau, \lambda}(x, x-y, \xi)= & \int e^{i\langle x-y, \eta\rangle} \partial_{\xi}^{\gamma-\mu-\lambda}\left\{\left[1-\chi_{j}\left(\frac{\eta}{|\xi|}\right)\right]\right. \\
& \left.\times\left[1-\chi_{j}\left(\frac{\xi+\eta}{|\xi|}\right)\right] a_{(\tau)}^{(\mu)}(x, \xi+\eta)\right\} \eta^{\alpha}|\eta|^{-2 N^{\prime}-2[\delta|\tau| / 2]} d \eta .
\end{aligned}
$$

Observing that the support of the integrand is in the domain $|\eta| \geqq|\xi| / 4$ and $|\xi+\eta| \geqq|\xi| / 4$, we have the estimate

$$
\left|H_{\alpha, \mu, \tau, \lambda}(x, x-y, \xi)\right| \leqq C^{N+|\gamma-\lambda|+|\tau|+1}|\gamma-\lambda| ! \cdot \tau !^{\sigma}|\xi|^{-N-\rho|\gamma-\lambda|},
$$

where the constant $C$ is independent of $N, \mu$ and $\tau$. On the other hand, since we have taken $l=N+|\beta|$ and $\tau \leqq \beta,|\alpha|=N^{\prime}$, we have an estimate of type

$$
\begin{aligned}
\left|\left(-\Delta_{y}\right)^{[\delta|\tau| / 2]} u_{(\alpha+\beta-\tau)}^{(\lambda)}(y, \xi)\right| \leqq & C_{0} C_{1}^{N+|\lambda|+|\beta-\tau|} N !^{\sigma} \lambda !(\beta-\tau) !^{\sigma} \\
& \times[\delta|\tau|] !^{\sigma}|\xi|^{\left.m^{\prime \prime}-\rho|\lambda|+\delta(N+\mid \beta-\tau)+\delta|\tau|\right)} .
\end{aligned}
$$

From these estimates we have

$$
\begin{aligned}
\left|D_{x}^{\beta} \partial_{\xi}^{\gamma} I_{3}\right| \leqq C_{0} C_{1}^{N+|\gamma+\beta|} N !^{\sigma} \gamma ! \beta !^{\sigma}|\xi|^{m^{\prime \prime}-(1-\delta) N-\rho|\gamma|} & \\
& \times \sum_{\tau \leqq \beta}\left(\begin{array}{c}
\beta \\
\tau
\end{array}\right)[\delta|\tau|] !^{\sigma}|\xi|^{\delta|\beta-\tau|+\delta^{2}|\tau|} .
\end{aligned}
$$

Finally we shall consider $I_{4}(x, \xi)$. We rewrite

$$
\begin{aligned}
I_{4}(x, \xi)= & (2 \pi)^{-n} \iint e^{i\langle x-y, \eta\rangle} a(x, \xi+\eta)\left[h_{l}(y)-h_{k}(y)\right] b(y, \xi) d y d \eta \\
& -(2 \pi)^{-n} \iint e^{i\langle x-y, \eta\rangle} a(x, \xi+\eta)\left[h_{l}(y)-h_{k}(y)\right] b(y, \xi) \chi_{j}\left(\frac{\eta}{|\xi|}\right) d y d \eta \\
& -(2 \pi)^{-n} \iint e^{i\langle x-y, \eta\rangle} a(x, \xi+\eta)\left[h_{l}(y)-h_{k}(y)\right] b(y, \xi) \\
& \times\left[1-\chi_{j}\left(\frac{\eta}{|\xi|}\right)\right]\left[1-\chi_{j}\left(\frac{\xi+\eta}{|\xi|}\right)\right] d y d \eta \\
+(2 \pi)^{-n} \iint e^{i\langle x-y, \eta\rangle} a(x, \xi+\eta) h_{k}(y) b(y, \xi)\left[1-\chi_{j}\left(\frac{\eta}{|\xi|}\right)\right] & \times\left[1-\chi_{j}\left(\frac{\xi+\eta}{|\xi|}\right)\right] d y d \eta \\
& \quad \\
\equiv & I_{4,1}-I_{4,2}-I_{4,3}+I_{4,4} .
\end{aligned}
$$


We can easily see that $I_{4,1}(x, D)$ is an integral operator with kernel in $G_{x, z}^{\theta, 1 / \rho}$ in $\Omega^{\prime} \times \Omega^{\prime}$ uniformly with respect to $l, k \in Z_{+}$(cf. (2.4)).

Next we have

$$
\begin{aligned}
D_{x}^{\beta} \partial_{\xi}^{\gamma} I_{4,2}(x, \xi)=(2 \pi)^{-n} \sum_{\substack{\tau \leq \beta \\
\mu \leq \gamma}}\left(\begin{array}{c}
\beta \\
\tau
\end{array}\right)\left(\begin{array}{c}
\gamma \\
\mu
\end{array}\right) \iint e^{i\langle x-y, \eta\rangle}\left(1+|\eta|^{2}\right)^{-q} \eta^{\beta-\tau} \\
\quad \times a_{(\tau)}^{(\mu)}(x, \xi+\eta)\left(1-\Delta_{y}\right)^{q} \partial_{\xi}^{\gamma-\mu}\left\{\chi_{j}\left(\frac{\eta}{|\xi|}\right)\left[h_{l}(y)-h_{k}(y)\right] b(y, \xi)\right\} d y d \eta .
\end{aligned}
$$

By taking $q=q(\tau)=[(|\beta-\tau|+N) / 2]$, we have an estimate of type

$$
\begin{array}{r}
\left|D_{x}^{\beta} \partial_{\xi}^{r} I_{4,2}(x, \xi)\right| \leqq C_{0} C_{1}^{N+|\beta+\gamma|} N !^{\sigma} \gamma ! \beta !^{\sigma}|\xi|^{m_{+}+n-(\rho-\delta) N-\rho|\gamma|+\delta|\beta|}, \\
x \in \Omega^{\prime}, \quad|\xi| \geqq B .
\end{array}
$$

We can handle $I_{4,3}(x, \xi)$ similarly as in $I_{3}(x, \xi)$. It remains to consider $I_{4,4}$. We have

$$
\begin{aligned}
D_{x}^{\beta} I_{4,4}(x, \xi)=(2 \pi)^{-n} & \sum_{\tau \leqq \beta}\left(\begin{array}{c}
\beta \\
\tau
\end{array}\right) \iint e^{i\langle x-y, \eta\rangle}\left(1+|\eta|^{2}\right)^{-r} \eta^{\beta-\tau} a_{(\tau)}(x, \xi+\eta) \\
& \times \chi_{j}\left(\frac{\xi+\eta}{|\xi|}\right)\left[1-\chi_{j}\left(\frac{\eta}{|\xi|}\right)\right]\left(1-\Delta_{y}\right)^{r}\left\{h_{k}(y) b(y, \xi)\right\} d y d \eta .
\end{aligned}
$$

Taking $r=[(|\beta-\tau|+\delta|\tau|+s+n+2) / 2(1-\delta)]$ depending on $\tau$ and $s=0,1, \cdots$, and taking $k=2 r$, we have the estimate

$$
\begin{array}{r}
\left|I_{4,4(\beta)}(x, \xi)\right| \leqq C_{0} C_{1}^{s+|\beta|}(s+|\beta|) !^{\sigma /(1-\delta)}|\xi|^{-8-(n+1)}, \\
x \in \Omega^{\prime}, \quad|\xi| \geqq B, \quad s \in Z_{+} .
\end{array}
$$

By virtue of (2.9), the kernel of $I_{4,4}(x, D)$ given by

$$
I_{4,4}(x, z)=(2 \pi)^{-n} \int e^{i\langle x-z, \xi\rangle} I_{4,4}(x, \xi) d \xi
$$

is in $G^{\theta}\left(\Omega_{x}^{\prime} \times \Omega_{z}^{\prime}\right), \theta=\max (1 / \rho, \sigma /(1-\delta))$. Indeed, we have

$$
D_{x}^{\alpha} D_{x}^{\gamma} I_{4,4}(x, z)=(2 \pi)^{-n} \sum_{\beta \leq \alpha}\left(\begin{array}{l}
\alpha \\
\beta
\end{array}\right) \int e^{i\langle x-y, \xi\rangle} \xi^{\alpha-\beta+\gamma} I_{4,4(\beta)}(x, \xi) d \xi
$$

and we have the estimate (taking $s=|\alpha-\beta+\gamma|$ )

$$
\left|D_{x}^{\alpha} D_{z}^{\gamma} I_{4,4}(x, z)\right| \leqq C_{0} C_{1}^{|\alpha+\gamma|}|\alpha+\gamma| !^{\sigma /(1-\delta)}, \quad(x, z) \in \Omega^{\prime} \times \Omega^{\prime},
$$

where the constants $C_{0}$ and $C_{1}$ are independent of $\alpha, \gamma \in Z_{+}^{n}$. Summing up, we can split the operator as $F^{N}(x, D)=F_{1}^{N}+F_{2}^{N}$, where $F_{1}^{N}$ is an integral operator from $G^{\theta}\left(\Omega^{\prime}\right)$ into $G^{\theta}\left(\Omega^{\prime}\right)$ and $F_{2}^{N}$ is a pseudodifferential operator with symbol satisfying the condition $(\mathrm{F})$. By the above argument the property $\left(F^{\prime}\right)$ is obviously verified. 


\section{Gevrey hypoellipticity.}

THEOREM 3.1. (cf. [3, Theorem 3.1].) Let $a(x, \xi) \in S_{\rho, \delta, \sigma}^{m}\left(\Omega \times R^{n}\right)$ (see Definition 1.2), and assume that there are positive constants $c$ and $B$ and $-\infty<m^{\prime}<\infty$ such that

$$
|a(x, \xi)| \geqq c|\xi|^{m^{\prime}}, \quad x \in \Omega, \quad|\xi| \geqq B .
$$

Assume also that for any compact set $K \subset \Omega$, there are positive constants $C_{0}$ and $C_{1}$ such that

$\left(\mathrm{H}_{2}\right) \quad\left|a_{(\beta)}^{(\alpha)}(x, \xi)\right| \leqq C_{0} C_{1}^{|\alpha+\beta|} \alpha ! \beta !^{\sigma}|a(x, \xi)||\xi|^{-\rho|\alpha|+\delta|\beta|}, \quad x \in K, \quad|\xi| \geqq B$.

Then the operator $a(x, D)$ is Gevrey hypoelliptic of order $\theta, \theta=\max (1 / \rho$, $\sigma /(1-\delta))$, that is, if $u \in \mathscr{E}^{\prime}(\Omega)$ and $a(x, D) u$ is in $G^{s}$ in $\Omega^{\prime}, \Omega^{\prime} \subset \Omega$, then $u$ is also in $G^{s}$ in $\Omega^{\prime}$ for $s \geqq \theta$.

For the proof we need two lemmas. We first define the symbol of a left parametrix of $a(x, D)$ as usual by:

$$
\begin{gathered}
b_{0}(x, \xi)=1 / a(x, \xi), \quad x \in \Omega, \quad|\xi| \geqq B, \\
b_{j}(x, \xi)=-b_{0}(x, \xi) \sum_{1 \leqq|\alpha| \leqq j} b_{j-|\alpha|}^{(\alpha)} a_{(\alpha)}(x, \xi), \quad x \in \Omega,|\xi| \geqq B, j=1,2, \cdots .
\end{gathered}
$$

Take a function $\chi(\xi) \in C^{\infty}\left(R^{n}\right)$ such that $\chi(\xi)=0$ for $|\xi| \leqq B$ and $\chi(\xi)=1$ for $|\xi| \geqq B+1$, and set

$$
b^{N}(x, \xi) \equiv \chi(\xi) \sum_{j=0}^{N} b_{j}(x, \xi) .
$$

Then we have $b^{N}(x, \xi) \in S_{\rho, \delta, \sigma}^{m^{\prime}}\left(\Omega \times R^{n}\right)$.

LEMMA 3.1. Let $\Omega^{\prime}$ be a relatively compact open subset of $\Omega$, and take a function $h \in C_{0}^{\infty}(\Omega)$ such that $h=1$ in a neighborhood of $\bar{\Omega}^{\prime}$. Then we have

$$
\begin{aligned}
b^{N}(x, D) h a(x, D) & =I+R^{N}(x, D) \text { in } \Omega^{\prime}, \\
R^{N}(x, D) & =R_{1}^{N}+R_{2}^{N},
\end{aligned}
$$

where $R_{1}^{N}$ is an integral operator from $C^{\infty}\left(\bar{\Omega}^{\prime}\right)$ into $G^{\theta}\left(\bar{\Omega}^{\prime}\right)$ with kernel $R_{1}^{N}(x, y)$ and $R_{2}^{N}$ is a pseudodifferential operator with symbol $R_{2}^{N}(x, \xi)$ satisfying the conditions $(\mathrm{F})$ and $\left(\mathrm{F}^{\prime}\right)$ in Theorem 2.1.

Proof. By Theorem 2.1 we have

$$
b^{N}(x, D) h a(x, D)=r^{N}(x, D)+F^{N} \text { in } \Omega^{\prime},
$$

where the symbol of $r^{N}(x, D)$ is given by

$$
r^{N}(x, \xi)=\sum_{k=0}^{N} \sum_{|\alpha|=k} \frac{1}{\alpha !} \partial_{\xi}^{\alpha}\left(\sum_{j=0}^{N} b_{j}(x, \xi)\right) a_{(\alpha)}(x, \xi), \quad x \in \Omega^{\prime}, \quad|\xi| \geqq B .
$$


By the definition of $b_{j}(x, \xi), j=0,1, \cdots$, we have

$$
\begin{gathered}
r^{N}(x, \xi)=1+r^{N}(x, \xi)^{\prime}, \quad x \in \Omega^{\prime}, \quad|\xi| \geqq B, \\
\chi(\xi) r^{N}(x, \xi)^{\prime} \in S_{\rho, \delta, \sigma}^{m_{+}-(\rho-\delta) N}\left(\Omega^{\prime} \times R^{n}\right) .
\end{gathered}
$$

All the symbols of the class $S_{\rho, \delta}^{m_{+}-(\rho-\delta) N}\left(\Omega^{\prime} \times R^{n}\right)$ satisfy the conditions (F) and $\left(F^{\prime}\right)$. Hence we have the assertion of Lemma 3.1.

LEMMA 3.2. Let $R_{2}^{N}(x, y)$ be the kernel of the operator $R_{2}^{N}(x, D)$ given in Lemma 3.1. Then

$$
R_{2}^{N}(x, y) \in G_{x, y}^{\theta, 1 / \rho}\left(\Omega^{\prime} \times \Omega^{\prime} \backslash \Delta\right), \quad \Delta=\left\{(x, x) ; x \in \Omega^{\prime}\right\},
$$

where $\theta=\max (1 / \rho, \sigma /(1-\delta))$.

Proof. For simplicity we assume $N$ is so large that $N \geqq\left(m_{+}+n+2\right) /$ $(\rho-\delta)$. Let $U$ be a relatively compact open subset of $\Omega^{\prime} \times \Omega^{\prime} \backslash \Delta$. We shall estimate

$$
\sup _{U}\left|D_{x}^{\alpha} D_{y}^{r} R_{2}^{N}(x, y)\right| \text {. }
$$

By virtue of the construction of $R^{N}$ and by the fact $\theta \geqq \theta_{0}$, as in the proof of Theorem 1.1 the problem is reduced to estimating each term of the form

$$
\begin{aligned}
& I=\int e^{i\langle x-y, \xi\rangle} \xi^{\alpha-\beta}(-\xi)^{\gamma} u^{\tau}(x, \xi) d \xi \\
& =\left(x_{i}-y_{i}\right)^{-\left[\left(|\alpha-\beta|+|\gamma|+\delta|\beta-\tau|+\delta^{2}|\tau|\right) / \rho\right]} \int e^{i\langle x-y, \xi\rangle} \\
& \quad \quad \times D_{\xi_{i}}^{\left[\left(|\alpha-\beta|+|\gamma|+\delta|\beta-\tau|+\delta^{2}|\tau|\right) \rho\right]}\left\{\xi^{\alpha-\beta}(-\xi)^{\gamma} u^{\tau}(x, \xi)\right\} d \xi,
\end{aligned}
$$

for a fixed $i, 1 \leqq i \leqq n$ and $\tau \leqq \beta \leqq \alpha$.

By using the property $\left(F^{\prime}\right)$, we have an estimate of the form

$$
|I| \leqq C_{0} C_{1}^{N+|\alpha+\beta|} N !^{\sigma} \gamma !^{1 / \rho}(\alpha-\beta) !^{1 / \rho} \beta !^{\sigma} \tau !^{\sigma \delta}(\beta-\tau) !^{\delta / \rho} \tau !^{\delta^{2} / \rho} .
$$

For the right hand side of (3.3) we have an estimate of type

$$
(\alpha-\beta) !^{1 / \rho} \beta !^{\sigma} \tau !^{\sigma \delta}(\beta-\tau) !^{\delta / \rho} \tau !^{\delta^{2} / \rho} \leqq C^{|\alpha|}(\alpha-\tau) !^{\theta_{1}} \tau !^{\left(\sigma+\sigma \delta+\delta^{2} / \rho\right)} .
$$

Observing that we have

$$
\theta=\max (1 / \rho, \sigma /(1-\delta)) \geqq 1 / \rho, \sigma+\delta / \rho, \sigma+\sigma \delta+\delta^{2} / \rho .
$$

The last term is estimated by $C^{|\alpha|} \alpha !^{\theta}$. Thus we obtain an estimate of the form

$$
\sup _{U}\left|D_{x}^{\alpha} D_{y}^{r} R_{2}^{N}(x, y)\right| \leqq C_{0} C_{1}^{|\alpha+\gamma|} \alpha !^{\theta} \gamma !^{1 / \rho} .
$$

We remark that we must take $l=|\alpha|+N$ in the construction of $R_{2}^{N}$. 
However $R_{1}^{N}(x, y)$ is in $G^{\theta}$ in $\Omega^{\prime} \times \Omega^{\prime}$ uniformly with respect to $l, l=$ $0,1, \cdots$, and so we may use the operator $R_{2}^{N}$ as if it is not depending on $l$.

Proof of Theorem 3.1. We are considering the equation

$$
a(x, D) u=f, \quad u \in \mathscr{E}^{\prime}(\Omega), \quad f \in \mathscr{D}^{\prime}(\Omega),
$$

where $f$ is assumed to be $G^{s}$ in $\Omega^{\prime} \subset \subset \Omega, s \geqq \theta$. For simplicity we shall prove the case where $s=\theta=\max (1 / \rho, \sigma / 1-\rho)$. It is well known (cf. [4]) that $u$ is in $C^{\infty}\left(\Omega^{\prime}\right)$ under the hypotheses of Theorem 3.1. Now take an arbitrary point $x_{0} \in \Omega^{\prime}$ and a small neighborhood $U_{d}=\left\{x:\left|x-x_{0}\right|<d\right\} \subset \Omega^{\prime}$, $d>0$. Let $\varphi \in C_{0}^{\infty}\left(U_{d}\right)$ be such that $\varphi(x)=1$ on $U_{d / 2}$. Then we have by Theorem 1.1

$$
a(x, D) \varphi u=f-a(x, D)(1-\varphi) u \equiv f_{1} \in C^{\infty}\left(\Omega^{\prime}\right) \cap G^{\theta}\left(U_{d / 2}\right) .
$$

Next take $N$ sufficiently large so that $N \geqq\left(m_{+}+n+2\right) /(\rho-\delta)$, and take $h \in C_{0}^{\infty}(\Omega)$ so that $h=1$ on $\Omega^{\prime}$. Then by Lemma 3.1 , we have

$$
b^{N}(x, D) h a(x, D) \varphi u=\varphi u+R^{N}(x, D) \phi u=b^{N}(x, D) h f_{1} ;
$$

namely, we have an integral equation with respect to $\varphi u$ :

$$
\begin{aligned}
\varphi u & =b^{N}(x, D) h f_{1}-\int R_{1}^{N}(x, y) \varphi(y) u(y) d y-\int R_{2}^{N}(x, y) \varphi(y) u(y) d y \\
& \equiv g(x)-\int R_{2}^{N}(x, y) \varphi(y) u(y) d y,
\end{aligned}
$$

where $g(x)$ is a function in $G^{\theta}\left(U_{d / 2}\right)$. We set $R(x, y)=R_{2}^{N}(x, y)$ and denote its symbol by $R(x, \xi)$. Let $\omega=U_{d / 4}$ and assume $0<d \leqq 1$. We denote by $\omega_{\varepsilon}$ the open set of points in $\omega$ at distance $>\varepsilon$ from the complement of $\omega$ denoted by $\omega^{c}$. Then $\omega_{\varepsilon}=\varnothing$ if $\varepsilon>1 / 4$. We want to prove that there exists a constant $B$ such that for every $\varepsilon>0$ and every integer $j>0$ we have

$$
\varepsilon^{\theta|\alpha|} \sup _{\omega_{|\alpha| \varepsilon}}\left|D^{\alpha} u(x)\right| \leqq B^{|\alpha|+1} \quad \text { if } \quad|\alpha| \leqq j,
$$

and

$$
\varepsilon^{\theta k} \sup _{\omega_{k \varepsilon}}\left|(1-\Delta)^{[k / 2]} u(x)\right| \leqq B^{k+1} \quad \text { if } \quad k \leqq j .
$$

It follows from (3.6) or (3.6) that $u$ is in $G^{\theta}$ in $\omega$. Indeed, let $K$ be a compact subset of $\omega$ and choose $c>0$ so that $K \subset \omega_{c}$. Setting $j=|\alpha|$ and $\varepsilon=c /|\alpha|$ in (3.6), we obtain

$$
\sup _{K}\left|D^{\alpha} u\right| \leqq \sup _{\omega_{c}}\left|D^{\alpha} u\right| \leqq\left(B / c^{\theta}\right)^{|\alpha|+1}|\alpha|^{\theta|\alpha|},
$$

which proves that $u \in G^{\theta}(\omega)$. 
We shall prove (3.6) by induction on $j$. This is obviously true when $j=1$ if $B$ is sufficiently large. Assuming that (3.6) is proved for $j-1$, $j \geqq 2$, we shall show that (3.6) follows for $j$ if $B$ is sufficiently large and independent of $j$. To do so we only have to estimate the derivatives $D^{\alpha} u$ with $|\alpha|=j(j \geqq 2)$. Differentiation of (3.5) gives

$$
\varepsilon^{\theta j} \sup _{\omega_{j \varepsilon}}\left|D^{\alpha} u(x)\right| \leqq A^{j+1}+\varepsilon^{\theta j} \sup _{x \in \omega_{j \varepsilon}}\left|D_{x}^{\alpha} \int R(x, y) \varphi(y) u(y) d y\right|,
$$

where $A$ is a positive constant independent of $j$. By observing the construction of $R=R_{2}^{N}$ we have

$$
\begin{aligned}
D_{x}^{\alpha} \int R(x, y) \varphi(y) u(y) d y= & \sum_{0 \neq \beta \leq \alpha}\left(\begin{array}{l}
\alpha \\
\beta
\end{array}\right) \int K_{\beta}(x, y) D_{y}^{\alpha-\beta}(\varphi u) d y \\
& +\int R(x, y) D_{y}^{\alpha}(\varphi u) d y .
\end{aligned}
$$

By virtue of the property $\left(F^{\prime}\right)$ we have

$$
K_{\beta}(x, y)=\sum_{\tau \leq \beta}\left(\begin{array}{l}
\beta \\
\tau
\end{array}\right) \int e^{i\langle x-y, \xi\rangle} u^{\tau}(x, \xi) d \xi .
$$

First we shall treat the last term in (3.7). From the proof of Lemma 3.2 , we may assume that there is a constant $C>0$ such that

$$
\left|D_{y}^{\gamma}(x, y)\right| \leqq C^{|r|+1} \gamma !^{\theta}|x-y|^{(-|\gamma|+1) / \rho}, \quad(x, y) \in U_{d} \times U_{d} \backslash \Delta .
$$

For $|\alpha|=j$, rewrite $\alpha=\alpha^{\prime}+\alpha^{\prime \prime}$ with $\left|\alpha^{\prime}\right|=j-1,\left|\alpha^{\prime \prime}\right|=1$. We have

$$
\begin{aligned}
& \varepsilon^{\theta j} \sup _{\omega_{j \varepsilon}}\left|\int R(x, y) D_{y}^{\alpha}(\varphi(y) u(y)) d y\right|=\varepsilon^{\theta j} \sup _{\omega_{j \varepsilon}}\left|\int D_{y}^{\alpha^{\prime \prime}} R(x, y) D_{y}^{\alpha^{\prime}}(\varphi(y) u(y)) d y\right| \\
& \quad \leqq \varepsilon^{\theta j} c^{2} \sup _{\omega_{(j-1) \varepsilon}}\left|D^{\alpha^{\prime}} u\right|+\varepsilon^{\theta j} \sup _{x \in \varepsilon_{j}}\left|\int_{\omega_{(j-1) \varepsilon}^{c}} D_{y}^{\alpha^{\prime \prime}} R(x, y) D_{y}^{\alpha^{\prime \prime}}(\varphi(y) u(y)) d y\right| \\
& \quad \equiv I_{1}+I_{2} .
\end{aligned}
$$

By assumption we have

$$
I_{1} \leqq \varepsilon^{\theta} C^{\prime} C^{2} B^{j},
$$

where $C^{\prime}$ is a small constant depending on $\omega$. Denoting symbolically by $D_{y}^{k}$ the differentiation of order $k \geqq 0$, we have

$$
\begin{aligned}
I_{2} \leqq & \varepsilon^{\theta j} \sum_{k=1}^{j-1} \sup _{x \in \omega_{j \varepsilon}}\left|\int_{\partial \omega_{(j-k) \varepsilon}} D_{y}^{k} R(x, y) D_{y}^{j-k-1} u d S_{y}\right| \\
& +\varepsilon^{\theta j} \sum_{k=1}^{j-1} \sup _{x \in \omega_{j \varepsilon}}\left|\int_{\omega_{(j-k-1) \varepsilon} \backslash \omega_{(j-k) \varepsilon}} D_{y}^{k+1} R(x, y) D_{y}^{j-k-1} u(y) d y\right| \\
& +\varepsilon^{\theta j} \sup _{x \in \omega_{j \varepsilon}}\left|\int_{\omega^{c}} D_{y}^{\alpha} R(x, y) \varphi(y) u(y) d y\right| \\
\equiv & I_{2,1}+I_{2,2}+I_{2,3} .
\end{aligned}
$$


By the induction assumption and by using (3.8), we have

$$
I_{2,1} \leqq C_{\varepsilon} \varepsilon^{\theta} \sum_{k=1}^{j-1} C^{k+1} k !^{\theta} \varepsilon^{k \theta}(k \varepsilon)^{\{-k+1) / \rho} B^{j-k} \leqq \varepsilon^{\theta} C_{\varepsilon} C B^{j} \sum_{k=1}^{j-1}(C / B)^{k}
$$

Similarly we have

$$
I_{2,2} \leqq \varepsilon^{\theta} C^{\prime} C^{2} B^{j} \sum_{k=1}^{j-1}(C / B)^{k-1}
$$

and

$$
I_{2,3} \leqq \varepsilon^{\theta} C^{\prime} C^{j+1}
$$

Hence if we take $B$ greater than $2 C$ we have an estimate of the form

$$
I_{1}+I_{2} \leqq \varepsilon^{\theta} C_{\varepsilon}\left(C^{2} B^{j}+C^{j+1}\right), \quad|\alpha|=j .
$$

We want to apply the same method to estimate the first sum on the right hand side of (3.7). By virtue of the property $\left(\mathrm{F}^{\prime}\right)$ we have

$$
K_{\beta}(x, y)=\sum_{\tau \leq \beta}\left(\begin{array}{l}
\beta \\
\tau
\end{array}\right) \int e^{i\langle x-y, \xi\rangle} u^{\tau}(x, \xi) d \xi
$$

Setting

$$
K_{\beta}^{\tau}(x, y)=\int e^{i\langle x-y, \xi\rangle} u^{\tau}(x, \xi)\left(1+|\xi|^{2}\right)^{-\left(\hat{\jmath}|\beta-\tau|+\delta^{2}|\tau|\right) / 2} d \xi,
$$

we have the following estimate as in (3.8):

$$
\left|D_{y}^{\gamma} K_{\beta}^{\tau}(x, y)\right| \leqq C^{|\beta+\gamma|+1} \beta !^{\sigma} \gamma !^{\theta} \tau !^{\sigma \delta}|x-y|^{-(|\gamma|+1) / \rho}, \quad(x, y) \in U_{d} \times U_{d} \backslash \Delta .
$$

Our purpose is to estimate $\varepsilon^{\theta j} \times(3.7)$ in the form

$$
\varepsilon^{\theta j} \sum_{k=1}^{j}\left(\begin{array}{l}
j \\
k
\end{array}\right) \sum_{l=0}^{k}\left(\begin{array}{l}
k \\
l
\end{array}\right) \sup _{\substack{x \in \omega j \varepsilon \\
k=|\beta| \\
l=|\tau|}} \mid \int K_{\beta}^{\tau}(x, y) D_{y}^{j-k}\left(1-\Delta_{y}\right)^{\left(\hat{\delta}(k-l)+\delta^{2} l\right) / 2}(\varphi u) d y,
$$

where $j=|\alpha|$. As before, we have for $1 \leqq k \leqq j$ and $0 \leqq l \leqq k$,

$$
\begin{aligned}
&\left(\begin{array}{l}
j \\
k
\end{array}\right)\left(\begin{array}{l}
k \\
l
\end{array}\right) \sup _{x \in \omega_{j \varepsilon}}\left|\int K_{\beta}^{\tau}(x, y) D_{y}^{i-k}\left(1-\Delta_{y}\right)^{\left(\delta(k-l)+\delta^{2} l\right) / 2}(\varphi u) d y\right| \\
& \leqq\left(\begin{array}{l}
j \\
k
\end{array}\right)\left(\begin{array}{l}
k \\
l
\end{array}\right) C^{k+1} k !^{o} l !^{\sigma \delta} \sup _{\omega_{(j-1) \varepsilon}}\left|D^{j-k-1}\left(1-\Delta_{x}\right)^{\left(\delta(k-l)+\delta^{2} l\right) / 2} u(x)\right| \\
&+\left(\begin{array}{l}
j \\
k
\end{array}\right)\left(\begin{array}{l}
k \\
l
\end{array}\right) \sup _{x \in \omega_{j \varepsilon}}\left|\int_{\omega_{(j-1) \varepsilon}^{c}} D_{y} K_{\beta}^{\tau}(x, y) D_{y}^{j-k-1}\left(1-\Delta_{y}\right)^{\left(\delta(k-l)+\delta^{2} l\right) / 2}(\phi u) d y\right| \\
& \equiv I_{1, k, l}+I_{2, k, l} .
\end{aligned}
$$

By assumption we have 


$$
\varepsilon^{\theta j} I_{1, k, l} \leqq C^{\prime} C^{k+1} B^{j}\left(\begin{array}{l}
j \\
k
\end{array}\right)\left(\begin{array}{l}
k \\
l
\end{array}\right) k !^{\sigma} l !^{\sigma \delta} \varepsilon^{\theta((1-\delta) k+\delta(1-\delta) l)} B^{-(1-\delta) k-1+\delta(1-\delta) l} .
$$

Since $\varepsilon \leqq 1 / 4 j$, we have

$$
\begin{aligned}
\varepsilon^{\theta j} I_{1, k, l} & \leqq C_{\varepsilon} C^{k+1} B^{j} B^{-(1-\delta) k-1} \frac{j ! k !^{o} l !^{o \delta}}{(j-k) !(k-l) ! l !}\left(\frac{1}{4 j}\right)^{\theta((1-\delta) k+\delta(1-\delta) l)} B^{\delta(1-\delta) l} \\
& \leqq C_{\varepsilon} C^{k+1} B^{j} B^{-(1-\delta) k-1} B^{\delta(1-\delta) l} .
\end{aligned}
$$

Thus we have

$$
\varepsilon^{\theta j} \sum_{l=0}^{k} I_{1, k, l} \leqq C_{\varepsilon} C B^{j}\left[C / B^{(1-\delta) 2}\right]^{k} .
$$

Hence if $B^{(1-\delta)^{2}} \leqq 2 C$, we have

$$
\varepsilon^{\theta j} \sum_{k=1}^{j} \sum_{l=0}^{k} I_{1, k, l} \leqq C_{\varepsilon} C B^{j} .
$$

In the same way as we treated $I_{2}$, we have $I_{2, k, l}$

$$
\begin{aligned}
& \leqq\left(\begin{array}{l}
j \\
k
\end{array}\right)\left(\begin{array}{l}
k \\
l
\end{array}\right) \sum_{s=1}^{j-k-1} \sup _{\omega_{j \varepsilon}}\left|\int_{\partial \omega_{(j-1) \varepsilon}} D_{y}^{s} K_{\beta}^{\tau}(x, y) D^{j-k-8-1}\left(1-\Delta_{y}\right)^{\left[\left(\delta(k-l)+\delta^{2} l\right) / 2\right]} u(y) d S\right| \\
& +\left(\begin{array}{l}
j \\
k
\end{array}\right)\left(\begin{array}{l}
j \\
l
\end{array}\right) \sum_{s=1}^{j-k-1} \sup _{x \in \omega_{j \varepsilon}}\left|\int_{\omega_{(j-s-1) \varepsilon} \backslash \omega_{(j-s) \varepsilon}} D_{y}^{s} K_{\beta}^{\tau}(x, y) D_{y}^{j-k-s}\left(1-\Delta_{y}\right)^{\left[\left(\delta(k-l)+\delta^{2} l\right) / 2\right]} u(y) d y\right| \\
& +\left(\begin{array}{l}
j \\
k
\end{array}\right)\left(\begin{array}{l}
k \\
l
\end{array}\right) \sup _{x \in \omega_{j \varepsilon}}\left|\int_{\omega_{k \varepsilon}^{c}} D_{y}^{j-k} K_{\beta}^{\tau}(x, y)\left(1-\Delta_{y}\right)^{\left[\left(\delta(k-l)+\delta^{2} l\right) / 2\right]}(\varphi u) d y\right| \\
& \equiv I_{2, k, l}^{1}+I_{2, k, l}^{2}+I_{2, k, l}^{3} .
\end{aligned}
$$

We have as before

$$
\begin{aligned}
\varepsilon^{\theta j} I_{2, k, l}^{1} \leqq & C_{\varepsilon} C^{k+1} B^{j}\left(\begin{array}{l}
j \\
k
\end{array}\right)\left(\begin{array}{c}
k \\
l
\end{array}\right) B^{-(1-\delta) k} \cdot \varepsilon^{\left(k+1-\delta(k-l)+\delta^{2} l\right) \theta} \\
& \times B^{-\delta(1-\delta) l} k !^{\sigma} l !^{\sigma \delta} \sum_{\delta=0}^{j-k-1}(C / B)^{s} s !^{\theta} \varepsilon^{s \theta}(\varepsilon s)^{(-8+1) / \rho} \\
\leqq & C_{\varepsilon} C^{k+1} B^{j} B^{-(1-\delta)^{2} k}=C_{\varepsilon} C B^{j}\left[C / B(1-\delta)^{2}\right]^{k}
\end{aligned}
$$

Hence we have

$$
\varepsilon^{\theta j} \sum_{k=1}^{j} \sum_{l=0}^{k} I_{2, k, l}^{1} \leqq C_{\varepsilon} C B^{j},
$$

if $B^{(1-\delta)^{2}} \geqq 2 C$. In the same way, we have an estimate of the form

$$
\varepsilon^{\theta j} \sum_{k=1}^{j} \sum_{l=0}^{k}\left(I_{2, k, l}^{2}+I_{2, k, l}^{3}\right) \leqq 2 C_{\varepsilon} C B^{j}
$$


Combining the estimates (3.9), (3.12), (3.13) and (3.14) we have

$$
\varepsilon^{\theta j} \sup _{\omega_{j \varepsilon}}\left|D^{\alpha} u(x)\right| \leqq A^{j+1}+C_{\varepsilon} C\left((C+5) B^{j}+C^{j}\right) .
$$

Hence if we can take $B$ so large that

$$
A^{j+1}+C_{\varepsilon} C\left((C+5) B^{j}+C^{j}\right) \leqq B^{j+1}
$$

the proof of (3.6) is completed. This condition is fulfilled for every $j$ if $B \geqq \max \left(1,(2 C)^{1 /(1-\delta)^{2}}, 2 A, A+C_{\varepsilon} C(C+6)\right)$.

4. Examples. We consider the following differential operators:

$$
\begin{aligned}
& P_{1}=-x^{4} d^{2} / d x^{2}+1 \text { in }-\infty<x<\infty, \\
& P_{2}=x^{4}\left(\partial / \partial y-\partial^{2} / \partial x^{2}\right)+1 \text { in } R_{x, y}^{2} .
\end{aligned}
$$

We can easily verify that the characteristic polynomial $P_{1}(x, \xi)=x^{4} \xi^{2}+1$ satisfies the conditions $\left(\mathrm{H}_{1}\right)$ and $\left(\mathrm{H}_{2}\right)$ in Theorem 3.1 with $\sigma=\rho=1$ and $\delta=1 / 2$. Hence we have $\theta=2$. We have a solution

$$
v(x)= \begin{cases}x e^{-1 / x} & x>0 \\ 0 & x \leqq 0\end{cases}
$$

of the equation $P_{1} v(x)=0$ and $v(x)$ is in $G^{2}$ in any neighborhood of the origin of $R^{1}$.

We can also easily verify that $P_{2}(x, y ; \xi, \eta)=v^{4}\left(i \eta+\xi^{2}\right)+1$ satisfies $\left(\mathrm{H}_{1}\right)$ and $\left(\mathrm{H}_{2}\right)$ with $\sigma=1, \rho=1 / 2$ and $\delta=1 / 4$. Hence we have $\theta=2$ also for $P_{2}$. We have a solution of the equation $P_{2} u=0$ as a function expressed by $u(x, y)=v(x)$, where $v(x)$ is the function given above.

We remark that we have only $\theta=1 /(\rho-\delta)=4$ by the result of [3].

\section{REFERENCES}

[1] P. Bolley, J. Camus et G. Métivier, Régularité Gevrey et itérés pour une classe d'opérateurs hypoelliptiques, editrice universitaria levrotto \& Bella-Torino, 1984.

[2] A. Friedman, On the regularity of solutions of nonlinear elliptic and parabolic systems of partial differential equations, J. Math. and Mech. 7 (1958), 43-60.

[3] S. Hashimoto, T. Matsuzawa ET Y. MoRimoto, Opérateurs pseudodifférentiels et classes de Gevrey, Comm. in Partial Differential Equations 8 (12) (1983), 1277-1289.

[4] L. Hörmander, Pseudodifferential operators and hypoelliptic equations, Proc. Symp. Pure Math. 83 (1966), 129-209.

[5] L. HöRMANDER, Uniqueness theorems and wave front sets for solutions of linear differential equations with analytic coefficients, Comm. Pure Appl. Math. 24 (1971), 671-704.

[6] V. IfTimie, Opérateurs hypoelliptiques dans les espaces de Gevrey, Bull. de la Societé des Sciences Math. de Roumanie 27 (75) (1983), 317-333.

[7] C. IWASAKI, Gevrey-hypoellipticity and pseudodifferential operators on Gevrey class, Lecture Notes in Math. 1256, Springer-Verlag, Berlin, Heidelberg, New York, Tokyo, 
1986, 281-293.

[8] P. Krée ANd L. Boutet De Monvel, Pseudodifferential operators and Gevrey classes, Ann. Inst. Fourier 17 (1) (1967), 295-323.

[9] T. Matsuzawa, Partially hypoelliptic pseudodifferential operators, Comm. in Partial Differential Equations 9 (11) (1984), 1059-1084.

[10] T. Matsuzawa, Partial regularity and applications, Nagoya Math. J. 104 (1986), 133-143.

[11] G. MÉTIVIER, Analytic hypoellipticity for operators with multiple characteristics, Comm. in Partial Differential Equations 6 (1) (1981), 1-90.

[12] S. Mizohata, On asymptotic expressions of symbols and formal symbols, Lecture Notes at Kyôto Univ. (1985).

[13] L. Rodino, Gevrey hypoellipticity for a class operators with multiple characteristics, Astérisque 89-90, (1984), 249-262.

[14] K. TANIGUChI, On multi-products of pseudodifferential operators in Gevrey classes and its application to Gevrey hypoellipticity, Proc. Japan Acad. 61, Ser. A (1985), 291-293.

[15] F. TREVES, Introduction to Pseudodifferential and Fourier Integral Operators, Vol. 1, Plenum Press, New York and London, 1981.

[16] L. R. Volevič, Pseudodifferential operators with holomorphic symbols and Gevrey classes, Trans. of Moskow Math. Society 24 (1971), 43-68.

Department of Mathematics

Faculty OF Science

NAGOYA UNIVERSITY

NAGOYA 464

JAPAN 